\title{
Influence of single and dual mass flywheel usage in IC engines on the resonance behavior of the power transmission system
}

\author{
Emin Güllü̈ ${ }^{1}$, Abdurrahman Yılmaz ${ }^{2 *}$ and Hakan Gökdağ ${ }^{3}$ \\ 0000-0001-9019-7617, 0000-0002-1300-8728, 0000-0003-3070-6365
}

${ }^{1}$ Mechanical Engineering Department, Faculty of Engineering, Bursa Uludağ University, Bursa, 16059, Turkey

${ }^{2}$ Automotive Department, Zuhal Dörtçelik Şenipek Vocational High School, Bursa, 16010, Turkey

${ }^{3}$ Mechanical Engineering Department, Faculty of Engineering and Natural Sciences, Bursa Technical University, Bursa, 16310, Turkey

\begin{abstract}
In internal combustion engines, due to the transmission of torsional vibrations caused by the irregularity of the combustion process to the gearbox, resonance occurs especially at low engine speeds. Resonance causes noise in the power transmission system. Therefore, driving comfort reduces and additional loads on the gearbox arise. Nowadays, with the concern of fuel consumption, the manufacturers turn to engine designs that keep the engine's working range as wide as possible. In this study, the mathematical models of power transmission systems using single-mass and dual-mass flywheel were analyzed and both systems were compared in terms of engine speed at which resonance occurs. In addition, the effects of the design parameters such as the mass moment of inertia and spring stiffness on the resonance behavior of the power transmission system using dual mass flywheel were examined. It has been observed that the dual mass flywheel drops the speed which resonance occurs in the power transmission system far below engine idle speed so that the resonance speed is kept away from the engine's working range.
\end{abstract}

Keywords: Dual mass flywheel, resonance, power transmission system, vibration
* Corresponding author

Abdurrahman Yilmaz

yilmaz3426@gmail.com.tr

Adress: Automotive Department, Zuhal Dörtçelik Şenipek Vocational High School, Bursa, 16010, Turkey

Tel: +902243715499

Fax: +902243717689

Researh Article $\begin{array}{ll}\text { Received } & 05.10 .2019 \\ \text { Revised } & 16.12 .2019 \\ \text { Accepted } & 25.12 .2019\end{array}$

Doi: 10.30939/ijastech..629705

\section{Introduction}

The dual mass flywheel can generally be defined as a single mass flywheel divided into two parts. It consists of the first mass attached to the crankshaft and the second mass connected to the gearbox input shaft. The distributed assembly picture of the dual mass flywheel produced by Schaeffler-LuK firm is shown in Figure 1.

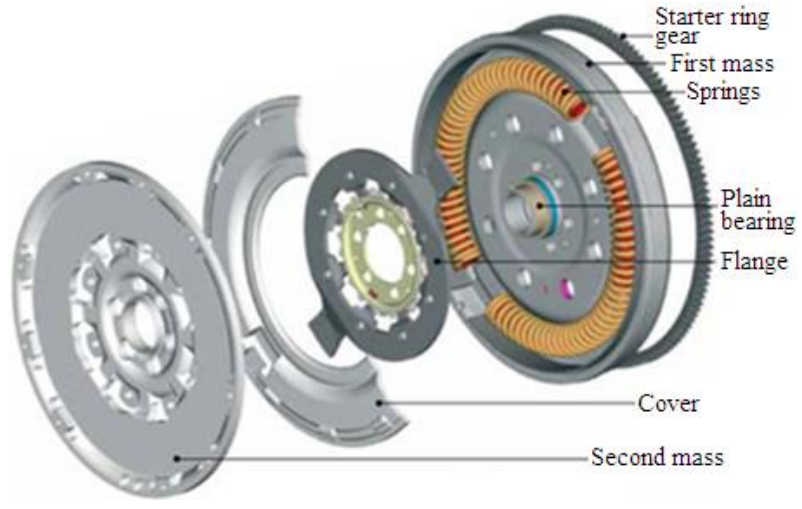

Fig. 1. Dual mass flywheel (Schaeffler-LuK) 
Two independent flywheels are interconnected by a springdamper system consisting of curved helical springs. These masses are supported by the bearing or sliding bearing to be rotatable in mutually opposite directions. On the first mass of the flywheel, there is a ring gear which corresponds to the starter gear. The first mass of the flywheel together with the first mass cap forms the gap required for the circular spring channel. The helical springs in the curved form fit into the guides in the spring channels. The motor torque is transmitted via the drive flange. The drive flange is firmly riveted to the second mass. The ears of flange fit between the spring channels of the first mass. The clutch system is located on the second mass of the flywheel.

There are torsional damping springs on the clutch disc in the mechanical clutch system to protect the gearbox and other powertrain components from torsional vibrations caused by speed irregularities in the engine. These springs are inadequate to damp the torsional vibrations and vibrations pass into the gearbox. This situation results in the resonance vibration, especially at low engine speeds. Resonance vibration causes noise and additional loads. Nowadays, with the concern of fuel consumption, the manufacturers turn to engine designs that keep the engine's working range as wide as possible. This situation requires a flicker-free operation at low engine speeds. The dual mass flywheel effectively damps torsional vibrations thanks to its spring damper system and drops the rpm at which the resonance occurs below idle speed.

There are many studies on the dual mass flywheel in literature. In particular, Schaeffler LuK's training notes and the articles written by the engineers in this company should be mentioned. This company is considered as the pioneer of dual mass flywheel technology and organizes the symposium on vehicle power transmission system technologies in every four years. In this company, the articles written by Schulte [1], Schnurr [2], Albers [3], Reik et al. [4] include the valuable information explaining the basic features and functions of the dual mass flywheel with clear pictures and graphics. Schulte [1] has made the first study describing the basic structure and functions of the dual mass flywheel. Schnurr [2] has made the first study examining the behavior of dual mass flywheel during engine cranking. Albers [3] has made a research on the modeling of dual mass flywheel torsion springs and flange in addition to summarizing the technological developments related to the system. Reik et al. [4] talked about innovations in the dual mass flywheel and investigated new methods for damping torsional vibrations in the powertrain. A book was prepared by Shaver [5] about the basic concepts of mechanical clutches in SAE. In this book, besides the effects of torsional vibrations on the gearbox, there is considerable information on the prevention of gear noises by damping the torsional vibrations of the dual mass flywheel. Chen et al. [6] made an experimental study to verify the vibration damping effect of the dual mass flywheel. They simulated working conditions such as idling, acceleration, and loading of an engine with the experimental setup they established. They showed that the vibration damping performance of the dual mass flywheel changed at different engine speeds and loads. As a result, they stated that the dual mass flywheel has a better damping effect at high engine speeds and loads. Chen et al. [7] developed a method of calculation called structural sensitivity analysis method. By using this method, they concluded the prime factors affecting the first-degree natural frequencies (resonance) of the powertrain have been the torsional stiffness and inertia of the dual mass flywheel. Meng and Li [8] made a study comparing the dual mass flywheel torsion spring and clutch disc torsion spring in damping torsional vibrations. They have created nine degrees of freedom models for both power transmission systems. They calculated the idle natural frequency and the idle natural vibration model using the generalized Jacobi algorithms and the compiled Matlab procedures. They conclude that the dual mass flywheel can protect the powertrain from idle resonance compared to the clutch disc. Mohire and Burde [9] made a study evaluating the interconnected behavior of the engine starter system and the dual mass flywheel. They stated that the speed of starter motor during the first run must be sufficient that the dual mass flywheel is passed from the resonance zone quickly otherwise the dual mass flywheel will be damaged. They have added that this problem can be caused by the inadequate charging of the vehicle battery. Gupta et al. [10] performed a theoretical and experimental study comparing the noise and vibration performance of a single mass flywheel, external vibration damper and dual mass flywheel as separate systems in a power transmission system of a rear wheel drive vehicle. The result is that using an external vibration damper together with the dual mass flywheel is a good solution and will increase the noise and vibration performance of the vehicle to acceptable levels. Yang et al. [11] have created a torsional vibration model to study the relationship between torsional vibrations and gear click noise occurring in the gearbox. This model incorporates the clutch system, the gearbox and the equivalent load of the entire vehicle in a front wheel drive vehicle. Using this model, they analyzed the effects of some parameters such as flywheel inertia, clutch rigidity and drive shaft stiffness on the formation of resonance at the gearbox input. They conclude that the optimized powertrain parameters reduce the resonance generation at the transmission input. Duran and Sever [12] analyzed the effect of the single and dual mass flywheel on the crankshaft fatigue safety factor for the various operating states of the engine using a special crankshaft dynamic simulation program.

In this study, mathematical model of power transmission systems using single and dual mass flywheels is solved in Matlab $^{\circledR}$ program, and resonance behavior of both systems 
is compared. In contrast to the studies in the literature, more detailed and clearer mathematical model was created, and the resonance status was examined according to different gear loads. Also, the effects of the change of some parameters in the dual mass flywheel were examined to resonance rpm.

\section{Equations of motion}

Two separate models have been created for power transmission systems using DMF and SMF. These models include flywheel, clutch system and gearbox and close parts (differential and axles for handling a front wheel drive). The power transmission system using SMF is shown in Figure 2.

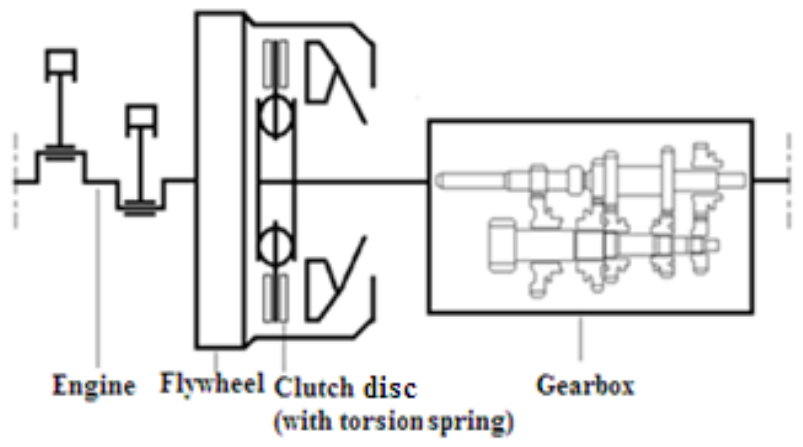

Fig. 2. Power transmission system using a single mass flywheel

The power transmission system using DMF is shown in Figure 3.

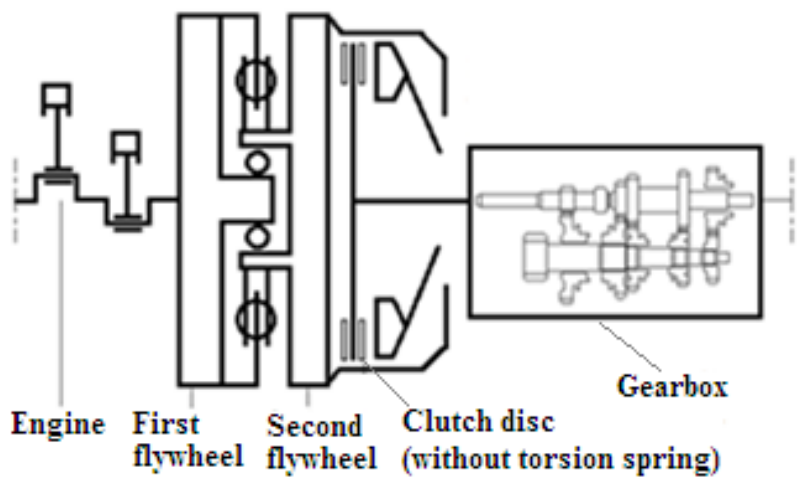

Fig. 3. Power transmission system using a dual mass flywheel

The clutch disc which used with SMF has torsion springs. On the other hand, a more rigid clutch disc without torsion springs is used with DMF. The spring system between two masses in DMF replaces the torsion springs in the clutch disc.

The mathematical model of the system using SMF is shown in Figure 4. This model is generated by using Figure 2 and the model of the power transmission system used in the study of Karadere et al. [13].

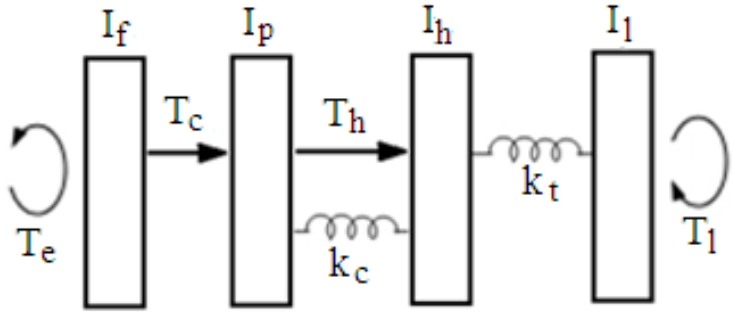

Fig. 4. The mathematical model of the system using a single mass flywheel

Similarly, the mathematical model of the system using DMF is shown in Figure 5.

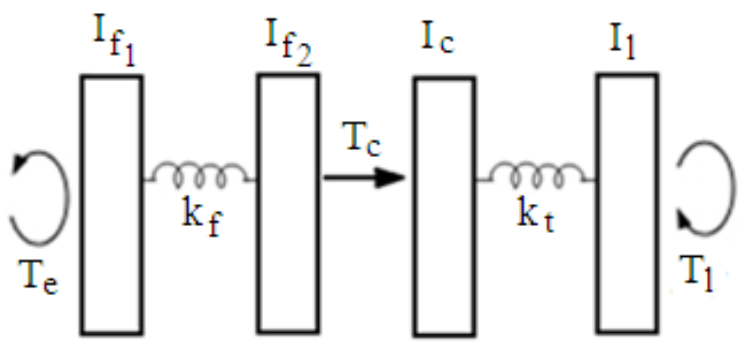

Fig. 5. The mathematical model of the system using a dual mass flywheel

In these models, the elements of the powertrain system are thought as discs connected by springs.

In these figures $T_{e}$ is the engine torque, $T_{c}$ is the torque due to the surface friction of the flywheel and the clutch disc, $T_{h}$ is the torque that is caused by friction elements placed between cushion spring plate and hub to suppress torsional vibrations. $T_{l}$ is load torque, i.e. the torque reduced to the clutch output of the powertrain components.

$I_{f}, I_{p}$ and $I_{h}$ denote the mass moments of inertia of the flywheel, clutch disc pad and disc hub in the classical powertrain system respectively. $I_{f 1}, I_{f 2}$ mean respectively the mass moments of inertia of the first and second mass of DMF in the powertrain system using DMF. $I_{c}$ is the mass moment of inertia of clutch disc in the powertrain system using DMF. $I_{l}$ is the mass moment of inertia reduced to the clutch output of the powertrain components. This parameter changes in different gears. $k_{t}$ is equivalent stiffness of the gearbox input shaft in both systems. $k_{c}$ is equivalent stiffness of torsion springs on clutch disc in classical powertrain system. $k_{f}$ represents equivalent stiffness of torsion springs in DMF.

For the solution, the unit moment will be applied to the input element (the first mass of the flywheel or flywheel) and the velocity ratio of the input and output elements will be obtained by observing the angular displacement behavior of the output element (powertrain i.e. load). For this purpose, the above models are rearranged without any other exciting moments except the exciting moment in the input element (flywheel or flywheel first mass). In this case, the revised mathematical 
model of the system using the single mass flywheel is shown in Figure 6. Since the clutch friction moment $T_{c}$ is disabled, the flywheel inertia and the clutch pad inertia are reduced to single inertia.

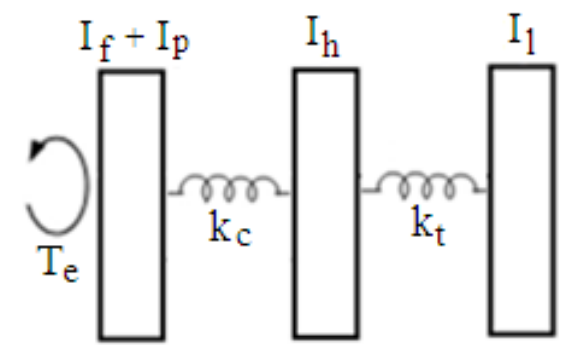

Fig. 6. Revised Mathematical model of the system using a single mass flywheel

Similarly, the revised mathematical model of the system using DMF is shown in Figure 7. Since the clutch friction moment $T_{c}$ is disabled, the second mass of flywheel inertia and clutch disc inertia are reduced to single inertia.

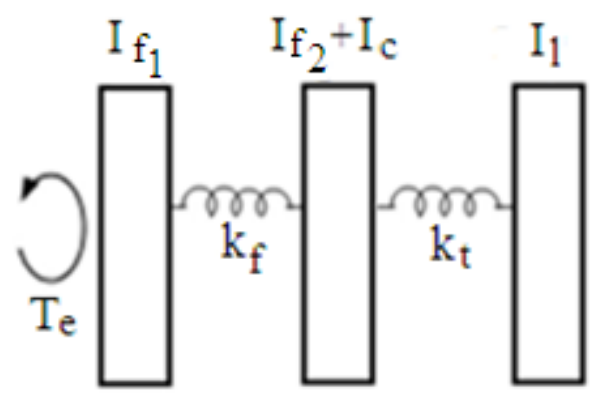

Fig. 7. Revised mathematical model of the system using a dual mass flywheel

From the revised model of the system using SMF, Newton's equations of motion of each element obtained as follows.

$I_{T} \ddot{q}_{T}+k_{c}\left(q_{T}-q_{h}\right)=T_{e}$

$I_{h} \ddot{q}_{h}-k_{c}\left(q_{T}-q_{h}\right)+k_{t}\left(q_{h}-q_{R}\right)=0$

$I_{\mathfrak{l}} \ddot{q}_{\mathfrak{R}}-k_{t}\left(q_{h}-q_{\mathfrak{R}}\right)=0$

In the first equation, the sum of the inertia of the flywheel and clutch disc pad is written as

$I_{T}=I_{f}+I_{p}$

Similarly, the equations of motions are obtained from the revised model of the system using DMF as follows.

$$
\begin{aligned}
& I_{f_{1}} \ddot{q}_{f_{1}}+k_{f}\left(q_{f_{I}}-q_{T}\right)=T_{e} \\
& I_{T} \ddot{q}_{T}-k_{f}\left(q_{f_{1}}-q_{T}\right)+k_{t}\left(q_{h}-q_{\mathbb{D}}\right)=0 \\
& I_{\mathbb{I}} \ddot{q}_{\mathbb{D}}-k_{t}\left(q_{T}-q_{\mathbb{R}}\right)=0
\end{aligned}
$$

In the fourth equation, the sum of the inertia of the clutch disc and the second mass of DMF is written as

$I_{T}=I_{f 2}+I_{c}$

The expression $q$ in these sets represents the angular displacements of elements in the above models.

The equation set of both systems is expressed in matrix form as follows.

$$
I \ddot{q}+K q=T
$$

When a harmonic input in the form of $\boldsymbol{T}=\underline{\boldsymbol{T}} e^{i \omega t}$ is applied to these systems having the general equations of motion as above, the response appears in the form of $\boldsymbol{q}=\boldsymbol{Q} e^{i \omega t}$ since no damping exists. $\boldsymbol{T}$ is the vector containing the amplitudes of the moments applied to each degree of freedom. $\boldsymbol{Q}$ is the response vector that contains the amplitudes of angular displacements. $\omega$ is the frequency parameter in $\mathrm{rad} / \mathrm{s}$ unit. Substituting these expressions in Eq. 7 leads to

$\left(\boldsymbol{K}-\omega^{2} \boldsymbol{I}\right) \boldsymbol{Q}=\underline{\boldsymbol{T}}$

Here, the $\boldsymbol{Q}$ response vector can be expressed as follows.

$Q=R \underline{T}$

Where the $\boldsymbol{R}$ matrix is defined as $\boldsymbol{R}=\left(\boldsymbol{K}-\omega^{2} \boldsymbol{I}\right)^{-1}$

An element of this matrix is the ratio of the $i^{\text {th }}$ response to the input applied to $\mathrm{j}^{\text {th }}$ freedom as

$R_{i j}=Q_{i} / \underline{T}_{j}$

When the moment input is applied to the first element according to Eq. 10, the angular displacement response of the third element is as follows.

$R_{31}=Q_{3} / \underline{T}_{1}$

Similarly, when the moment input is applied to the first element, the angular displacement response of the first element is as follows.

$R_{11}=Q_{1} / \underline{T}_{1}$

Accordingly, the ratio of angular displacements of the output (powertrain i.e. load) and input (engine) elements are expressed as follows. 


$$
Q_{3} / Q_{1}=R_{31} / R_{11}
$$

According to the angular displacement expression $\boldsymbol{q}=\boldsymbol{Q} e^{i \omega t}$, speeds are in $\boldsymbol{q}^{\prime}=i \omega \boldsymbol{Q} e^{i \omega t}$ form. If the speed intensities vector is defined as $\boldsymbol{\Omega}=\omega \boldsymbol{Q}$, then the rate of $\Omega_{3} / \Omega_{1}$ speeds will be equal to Eq.13 after the required simplifications.

According to the explanations above, the programs written in Matlab ${ }^{\circledR}$ for the mathematical models of both systems were run by entering the given parameters and the results shown in the next section were obtained.

\section{Numerical results}

In this section, the analysis results are obtained by using the following parametric data of a vehicle produced.

Table 1 shows the parametrical data of the system using SMF

Table 1. Parametric values of power transmission system using the single mass flywheel

\begin{tabular}{c|c|l}
\hline Inertia of flywheel & $I_{f}$ & $0,135 \mathrm{kgm}^{2}$ \\
\hline Inertia of clutch disc pad & $I_{p}$ & $0,04 \mathrm{kgm}^{2}$ \\
\hline Inertia of clutch disc hub & $I_{h}$ & $0,0024 \mathrm{kgm}^{2}$ \\
\hline $\begin{array}{c}\text { Stiffness of torsion springs on clutch } \\
\text { disc }\end{array}$ & $k_{c}$ & $367 \mathrm{Nm} / \mathrm{rad}$ \\
\hline
\end{tabular}

Table 2 shows the parametrical data of the system using DMF.

Table 2. Parametric values of power transmission system using the dual mass flywheel

\begin{tabular}{c|c|l}
\hline $\begin{array}{c}\text { Inertia of the first mass of the } \\
\text { dual mass flywheel }\end{array}$ & $I_{f l}$ & $0,09 \mathrm{kgm}^{2}$ \\
\hline $\begin{array}{c}\text { Inertia of the second mass of the } \\
\text { dual mass flywheel }\end{array}$ & $I_{f 2}$ & $0,045 \mathrm{kgm}^{2}$ \\
\hline $\begin{array}{c}\text { Inertia of clutch disc using with } \\
\text { dual mass flywheel }\end{array}$ & $I_{c}$ & $0,00624 \mathrm{kgm}^{2}$ \\
\hline $\begin{array}{c}\text { Stiffness of torsion springs in } \\
\text { the dual mass flywheel }\end{array}$ & $k_{f}$ & $68 \mathrm{Nm} / \mathrm{rad}$ \\
\hline
\end{tabular}

Table 3 shows the reduced mass inertia of the gearbox and other powertrain components to the clutch output in different gears.

Table 3. Reduced mass moment of inertia of the gearbox and other powertrain components to the clutch output

\begin{tabular}{c|l|l}
\hline Inertia of load for 1.gear & $I_{l l g}$ & $0,00715 \mathrm{kgm}^{2}$ \\
\hline Inertia of load for 2.gear & $I_{l 2 g}$ & $0,011 \mathrm{kgm}^{2}$ \\
\hline Inertia of load for 3.gear & $I_{l 3 g}$ & $0,016 \mathrm{kgm}^{2}$ \\
\hline Inertia of load for 4.gear & $I_{l 4 g}$ & $0,0258 \mathrm{kgm}^{2}$ \\
\hline Inertia of load for 5.gear & $I_{l 5 g}$ & $0,0423 \mathrm{kgm}^{2}$ \\
\hline
\end{tabular}

The horizontal axis of the graphs shows the engine speed. The vertical axis shows the speed ratio of the engine and the load (gearbox and other powertrains), i.e., the speed fluctuations. Figure 8 shows the resonance behavior of systems using single and dual mass flywheels at third gear load. It is noteworthy that DMF drops the resonance rpm considerably below the idle speed when compared to SMF.

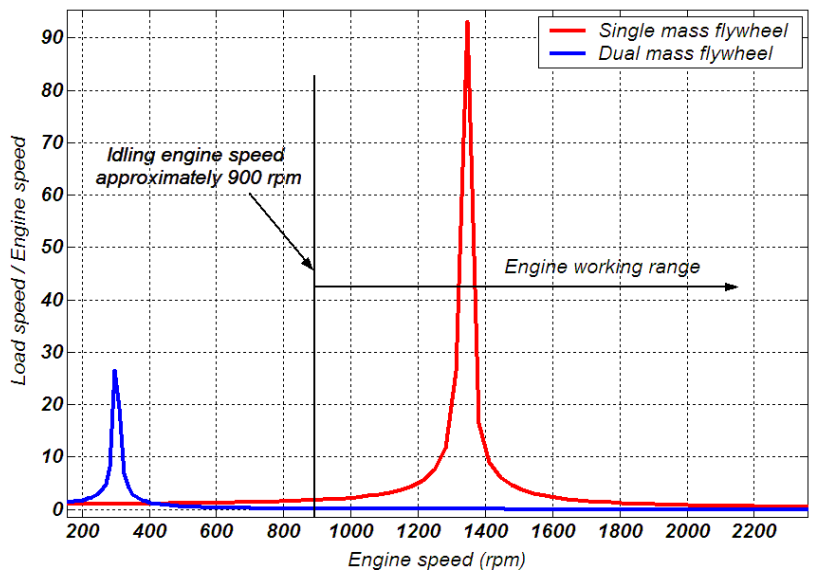

Fig. 8. Resonance behavior of systems using single and dual mass flywheels at third gear load.

Figure 9 shows the resonance behavior of the system using SMF according to different gear loads. It is seen that the resonance rpm's in the system using SMF remains within the motor operating range on other gear loads except 5 . gear.

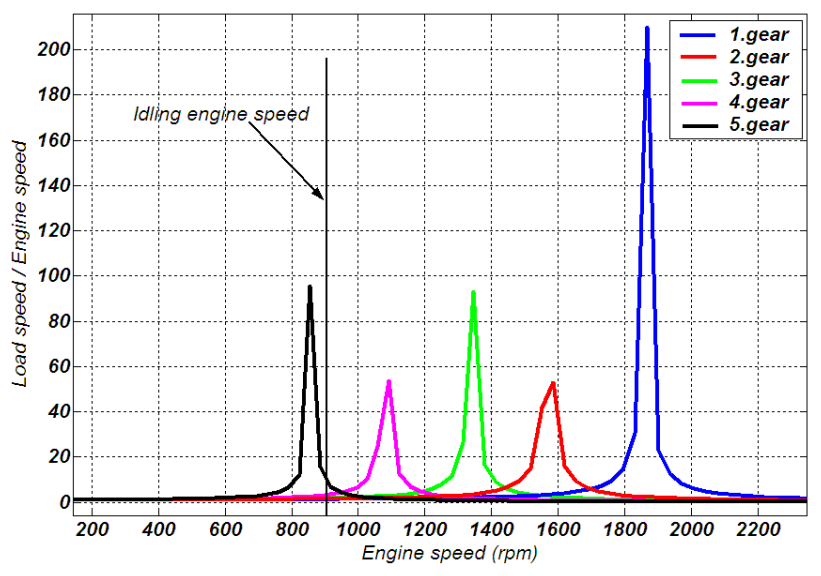

Fig. 9. Resonance behavior of the system using SMF for different gear load

Figure 10 shows the detailed graphics of the resonance behavior of the system using DMF according to different gear loads. It is seen that the resonance rpm's in the system using DMF is pulled out quite outside of the motor operating range (200- $400 \mathrm{rpm}$ ) on all gear loads. Resonance rpm's in gears is showed in extended graphic on Fig.10. 


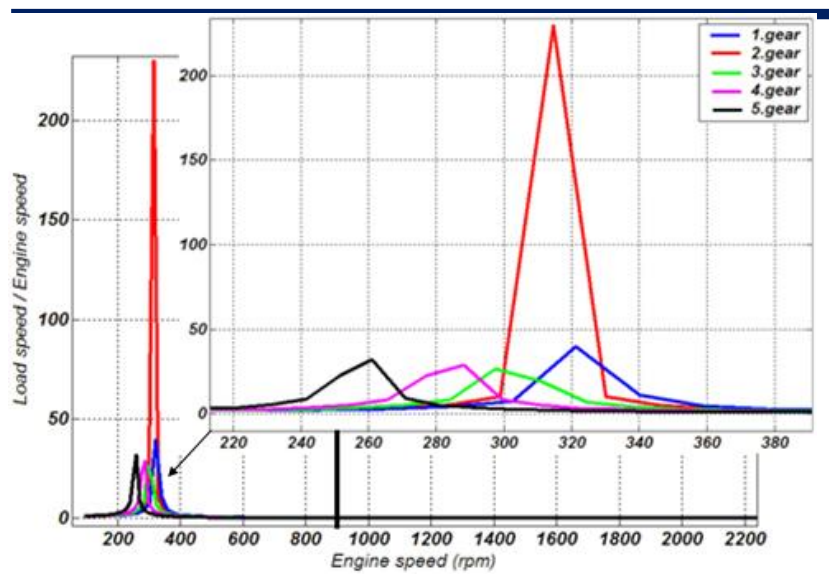

Fig.10. Resonance behavior of the system with DMF for different gear load

From the figures, it is understood that the increasing of load inertia drops the speed at which resonance occurs in both systems. However in the system with SMF, while the resonance rpm remains within the motor operating range DMF drops the resonance rpm to quite out of the idle speed.

Figure 11 shows the change of the resonance rpm for the different rigidity values of the torsion spring between the flywheel masses in the system using DMF. As the stiffness of the torsion spring decreases, the speed of resonance generation is reduced. Resonance rpm's for different rigidities is showed in extended graphic on Fig.11.

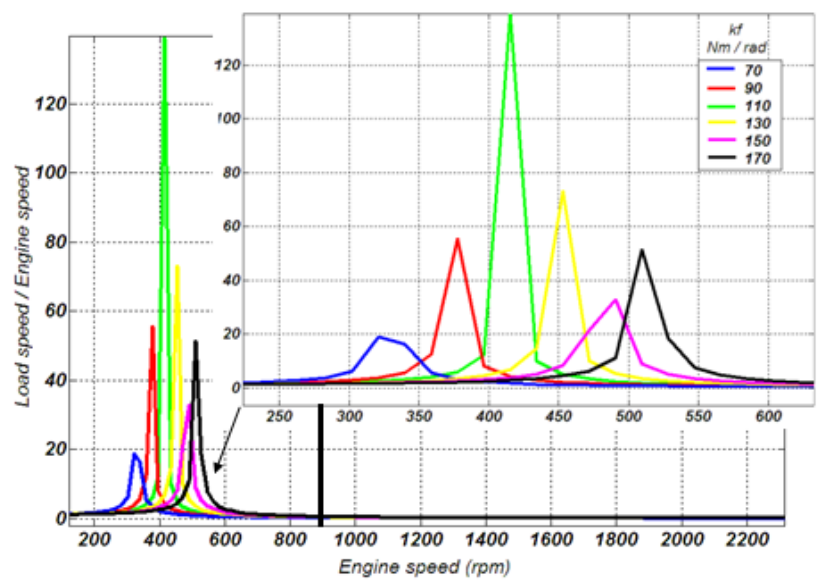

Fig.11. Variation of the resonance speed for different stiffness values of torsional spring of dual mass flywheel

DMF removes the resonance rpm well out of the engine's working range. Considering that the resonance rpm decreases as the stiffness of the springs decreases (Fig. 11), this is because the stiffness of DMF springs is quite small from the stiffness of the clutch disc springs ( $k_{c}=367 \mathrm{Nm} / \mathrm{rad}$ while $k_{f}$ $=68 \mathrm{Nm} / \mathrm{rad}$ ). Thanks to decreasing of spring stiffness, i.e., with the use of softer spring the resonance rpm has been reduced to quite out of idling rpm. It is understood that spring rigidity is an effective tool in decreasing the speed at which resonance occurs.

Figure 12 shows the change in the resonance speed at the same load and spring stiffness. It was assumed that the equivalent rigidity $\left(k_{c}\right)$ of the torsion springs in the clutch disc of the system using SMF is equal to the equivalent stiffness $\left(k_{f}\right)$ of the torsion springs between the flywheels in the system using DMF. It is seen although the difference between the resonance rpm of both systems is reduced at the same spring rigidity, the system using DMF was maintained its superiority.

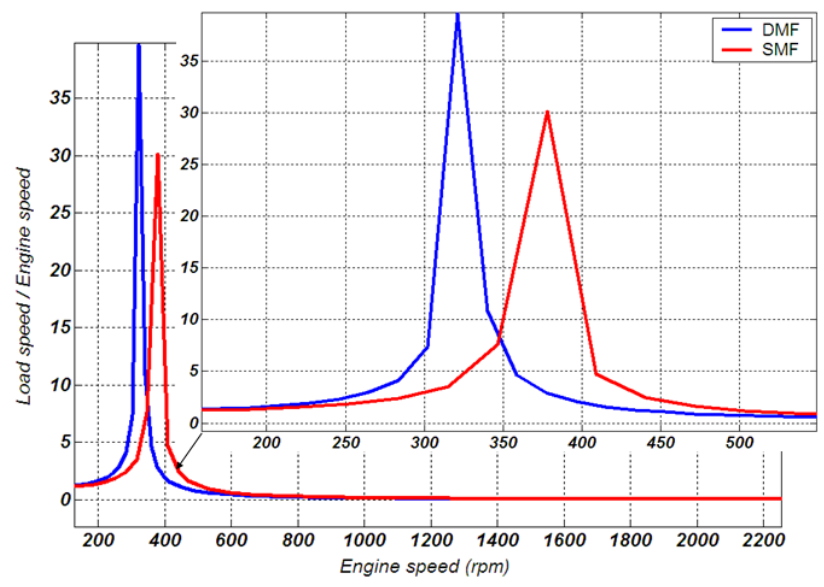

Fig. 12. Change of engine resonance rpm when both systems have the same load inertia and same spring stiffness $\left(k_{c}=k_{f}=68 \mathrm{Nm} / \mathrm{rad}, I_{l 2 g}=0,00715 \mathrm{kgm}^{2}\right)$

Figure 13 shows the change of the resonance rpm for the different ratios of the first and second masses of DMF. As the amount of the second mass of DMF increases, the speed at which the resonance occurs is dropped.

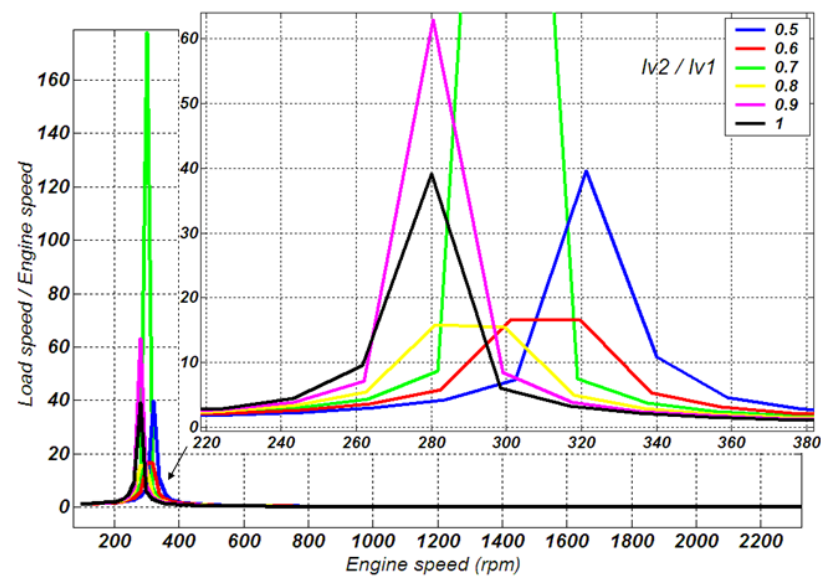

Fig. 13. Variation of engine resonance speed according to different ratios of the first and second masses of the dual mass flywheel

When the stiffnesses of the both systems are equal to each other, the resonance rpm of the system using SMF falls 
considerably below the idle speed, and the difference between the resonance rpm's is considerably reduced. Nevertheless, the system using DMF retained its superiority. Considering that the increasing of the inertia of DMF second mass drops the resonance rpm the reason for this is that the second mass of DMF increases the moment of inertia of the gearbox and the load. The inertia of the second mass of DMF is added to the inertia of the powertrain, i.e., the load. The increasing of load inertia drops the resonance rpm.

These findings that were explained until here support the results which were achieved from the studies [6], [7] and [8] numbered that have been mentioned in the introduction section

\section{Conclusions}

In this study, the effect of a single mass and dual-mass flywheel usage on the resonance behavior of the vehicle power transmission system were examined, and in summary, the following results were reached. The equivalent torsion spring stiffness of DMF is quite small compared to the equivalent stiffness of the torsion springs in the clutch disc in the conventional system. This is highly effective in dropping the resonance rpm. Although not as much as the reducing of spring stiffness, the increasing of the inertia of the second mass of DMF seems to be an effective feature in dropping the resonance rpm. Although the resonance amplitudes of the system using DMF also quite high, this may not be a problem as the resonance rpm is out of the engine working range except the cranking situation.

\section{Abbreviations}

SMF : Single mass flywheel

DMF : Dual mass flywheel

\section{References}

[1] Schulte, L. F. (1986). Dual Mass Flywheel. Proceedings of $3^{\text {th }}$ LuK Symposium.

[2] Schnurr, M. (1990). Development on the Super-Long-Travel DMFW. Proceedings of $4^{\text {th }}$ LuK Symposium.

[3] Albers, A. (1994). Advanced Development of DMFW Design - Noise Control for Today's Automobiles. Proceedings of $5^{\text {th }}$ LuK Symposium.

[4] Reik, W., Seebacher, R. and Kooy, A. (1998). The Dual Mass Flywheel. Proceedings of $6^{\text {th }}$ LuK Symposium.

[5] Shaver, J. (1997). Manual Transmission Clutch Systems. SAE Publications.

[6] Chen, D. M., Xu, J. H., Shi, X. F. and Ma, Y. Y. (2012). Experimental Study on Torsional Vibration of Dual Mass Flywheel. Advanced Materials Research, 490-495, 2318-2322.

[7] Chen, L., Zhong, H., Luo, X. and Zeng, R. (2018). Analysis Method of Structural Sensitivity of Dual Mass Flywheel and Power Transmission. IOP Conference Series: Materials
Science and Engineering, 5.Intelligent Manufacturing and Information Technology, 392.

[8] Meng, X. and Li, J. (2011). Idling Natural Characteristic Analysis of the Torsion Absorber with dual mass flywheel. Applied Mechanics and Materials, 80-81, 860-864.

[9] Mohire, S. and Burde, R. (2010). Evaluation of Interdependent Behavior of Dual Mass Flywheel (DMF) and Engine Starting System. SAE Technical Paper.

[10] Gupta, K., Choudhary, A. and Bidre, R. (2017). NVH Performance Improvement Study Using a Dual Mass Flywheel (DMF), Inertia Ring Type Tuned Torsional Vibration Damper (TVD) and Single Mass Flywheel (SMF) in a Front Engine and Rear Wheel Driveline Architecture. SAE Technical Paper.

[11] Yang, X., Pang, J., Wang L., Tian, X. and Tang, Y. (2017). A Study on Reducing Gear Rattle Noise Based on Sensitivity Analysis of Drivetrain Torsional Model. SAE Technical Paper.

[12] Duran, E. and Sever, A. (2008). Dynamic Simulation and Endurance Limit Safety Factor Calculation for Crankshaft Comparison of Single Mass and Dual Mass Flywheel. SAE Technical Paper.

[13] Karadere, G., Kopmaz, O. and Güllü, E. (2010). Transient phenomena during engagement phase of a clutch. International Journal of Materials and Product Technology, 39, 3/4, 225-239. 